\title{
Development of a Data Generator for Multivariate Numerical Data with Arbitrary Correlations and Distributions
}

\author{
Kai Vahldiek ${ }^{\mathrm{a}}$, Libing Zhou ${ }^{\mathrm{a}}$, Wenfeng Zhu ${ }^{\mathrm{a}}$ and Frank Klawonn ${ }^{\mathrm{a}, \mathrm{b} 1}$ \\ ${ }^{a}$ Department of Computer Science, Ostfalia University of Applied Sciences, D-38302 \\ Wolfenbüttel, Germany \\ ${ }^{\mathrm{b}}$ Helmholtz Centre for Infection Research, Biostatistics, Inhoffenstr. 7, D-38124 \\ Braunschweig, Germany
}

\begin{abstract}
Artificial or simulated data are particularly relevant in tests and benchmarks for machine learning methods, in teaching for exercises and for setting up analysis workflows. They are relevant when real data may not be used for reasons of data protection, or when special distributions or effects should be present in the data to test certain machine learning methods. In this paper a generator for multivariate numerical data with arbitrary marginal distributions and - as far as possible - arbitrary correlations is presented. The data generator is implemented in the open source statistics software R. It can also be used for categorical variables, if data are generated separately for the corresponding characteristics of a categorical variable. Additionally, outliers can be integrated. The use of the data generator is demonstrated with a concrete example.
\end{abstract}

Keywords. Data generator, data sets, correlations, distribution functions, simulations

\section{Introduction}

Machine learning and data mining belong to the field of explorative data analysis. In terms of content, correlations and patterns are found in data and prediction models are created. As shown in [1], a process model - such as the CRISP-DM or KDD model forms the basis for corresponding data analysis problems and projects. Machine learning offers numerous procedures, some of which pursue the same goals - for example, the detection of outliers or the creation of a prediction model - using different methods. There are many procedures that are suitable for the same or similar problems, and it is often not clear which procedure is best suited for a given data set. Very similar results can be achieved with different methods.

In the context of teaching, an application of the data generator is suitable. It is advisable to use certain data sets. The students should use the corresponding data sets to apply the learned methods and algorithms independently and comprehensibly. The data set must therefore guarantee certain properties to be examined. Furthermore, it must be variable in size. A modular structure consisting of several variables that can be combined

\footnotetext{
${ }^{1}$ Corresponding Author: Frank Klawonn, Department of Computer Science, Ostfalia University of Applied Sciences, Salzdahlumer Str. 46/48, D-38302 Wolfenbüttel, Germany; E-mail: f.klawonn@ ostfalia.de.
} 
with each other is advantageous. Thus, it should be possible to generate small and simple but also large and complex data sets with the data generator. Accordingly, a separate data set with specific properties can be generated for each test object during tests. However, the structural properties of all data sets remain the same.

The use of real data is often problematic because they are not available in sufficient quality or are too complex and ambiguous because they do not have the required characteristics. Therefore, artificial data with corresponding properties are used, which can be generated with the data generator. Relationships between individual variables or outliers can be included and the data can be generated according to specific distributions. This flexibility makes it possible to test the various machine learning algorithms using the generated data sets. Many existing data generators are only suitable for the application of certain algorithms. For each algorithm, a new special data set must be generated each time. In addition, there are only a few general data generators that take a holistic approach. Further use cases are the anonymization of real data for the creation of workflows, since the real data often may not be used for reasons of data protection law. For example, the data generator can also be used for benchmarking of clustering and classification algorithms.

The aim of the paper is to create a data generator for the generation of complex data sets with predefined properties. Multivariate numerical data with arbitrary marginal distributions are generated. The structure of the paper can be described as follows: First, related work and further approaches to possible data generators are presented. This is followed by the implementation and description of the methods and techniques used to generate special data sets according to predefined properties. Finally, various experiments in the context of the implementation as well as the practical application of the data generator are described based on an example.

\section{Related work}

As already mentioned, some data generators are available, but they are only suitable for concrete problems. For the application of certain algorithms, the corresponding data sets must always be generated accordingly. A general approach has not been followed so far.

For the analysis of clusters and outliers a data set generator was developed in [2]. It is based on an approach of different distribution and transformation functions to generate multidimensional numerical data sets. The user can specify various individual properties of the data set for later cluster analysis. However, this data generator only refers to the method of cluster analysis.

A further data generator in [3] is available for the evaluation of classification algorithms. The number of input features as well as the output classes to be generated can be set flexibly. Furthermore, the distribution of the data to be generated can be varied as well as the number of data sets. The data generator is called "DataGen". The data is generated either according to the classical normal distribution or the continuous equal distribution. Overall, the generator offers the possibility to compare the accuracy and speed of monitored classification algorithms with a uniform data set.

In [4] a benchmarking of classification algorithms for real data is presented, which is of great importance in applied data analysis. For the majority of the classification problems investigated, data preprocessing did not lead to an improvement of the performance of a computer cluster. Different preprocessing options are advantageous for different classifiers. Furthermore, some benefit more from preprocessing than other 
classifiers. At this point, a data generator is useful for limiting the problem when executing the algorithms, since the data records with the corresponding properties already exist, which can potentially improve performance. Nevertheless, there is a possibility that preprocessing can better take into account the effects of the distributions.

Data generators can also be used for classification problems. In [5] the effect of the correlation structure of independent variables on the discrimination of risk prediction models is investigated. Multivariate normally distributed data mean that zero correlation between predictive variables is often detrimental to discrimination in a risk prediction model, and negatively correlated predictive variables with positive effect sizes are beneficial. The data generator can generate a separate data set for each class, since the classification primarily considers intra-class correlations. These correlations can be defined by the generator.

Synthetic data sets are used in [6] for validation and benchmarking of machine learning algorithms. Special requirements for synthetic data are discussed and the generation and evaluation of these data is explained. Data protection should be guaranteed. In the end a case study illustrates the use of synthetic data.

In [7] a data synthesizer algorithm for categorical data is developed. The algorithm is special in that it uses high-dimensional categorical data. This data can be processed even if it is not provided as a contingency table. The algorithm is tested using sample data. Marginal and conditional distributions as well as regression coefficients from the synthetic data are compared with the original data.

Whether support vector machines can be used to develop synthetic data is investigated in [8]. Although the developed application is limited to categorical data, an extension to continuous data could be straightforward. Besides the general description of SVM, the prerequisites for the generation of synthetic data are also mentioned. Afterwards the algorithm is tested on a concrete example.

MDCGen is a tool for the generation of multidimensional synthetic data sets, which is presented in [9]. These data sets are used for testing, evaluation and benchmarking of unsupervised classification algorithms. Normal and non-normal distributions can be combined. During implementation, features such as cluster-separation and the addition of outliers and noise are considered.

In [10] a method for the simulation of multivariate ordinal variables is described. The ordinal variables have specified marginal distributions and correlation structures. Correlated binary variations are simulated. The calculation is performed in such a way that a reconversion to the ordinal scale provides the original distribution properties.

A modular, highly flexible open source environment for data generation is presented in [11]. Different types of modules for categorical and numerical data generators can be combined. The general environment as well as the individual modules for data generation will be presented using a concrete application example.

A concrete data generator, which is applicable for example for many algorithms of machine learning, is currently not available. In this paper a first approach is provided, which offers a high flexibility based on some individual settings for the generation of the data sets and can be applied in many areas. 


\section{Approach to realization}

The realization of the entire data generator consists of several parts. For each attribute of the data set any continuous distribution including mixed distributions can be specified. To model dependencies between the attributes, any correlation matrix can be defined. The classical correlation coefficient according to Pearson [12] is primarily intended for approximately normally distributed data and is extremely susceptible to outliers. Therefore, for our synthetic data the correlation matrix is specified using rank correlations according to Spearman [13] or Kendall [14]. The correlation matrix does not need to be fully specified, nor does it need to be consistent in the sense that there is actually a multivariate distribution with the specified correlations. The rank correlation values are first converted into correlation values according to Pearson. Then data of a multivariate normal distribution is to be generated that has at least approximately the same correlation matrix. If the correlation matrix is inconsistent, it is approximated by a suitable symmetrical, positive semidefinite matrix. Correlation coefficients that are not specified are not taken into account in the approximation. The generated multivariate data should then have approximately the specified rank correlation values. Then the individual normally distributed attributes are transformed into the specified distributions using inverse transformation. Since these transformations are monotonous, the rank correlation values do not change. In the following, the creation of a graphical user interface will also be discussed in the context of an example. The data generator offers a high degree of flexibility taking into account the individual properties of the data set to be generated.

\subsection{Generation of the data set}

The generation of the data set consists of seven steps. All steps are explained in detail in the following chapter and are then also considered in the graphical user interface in chapter 5.3. Figure 1 provides a first overview.

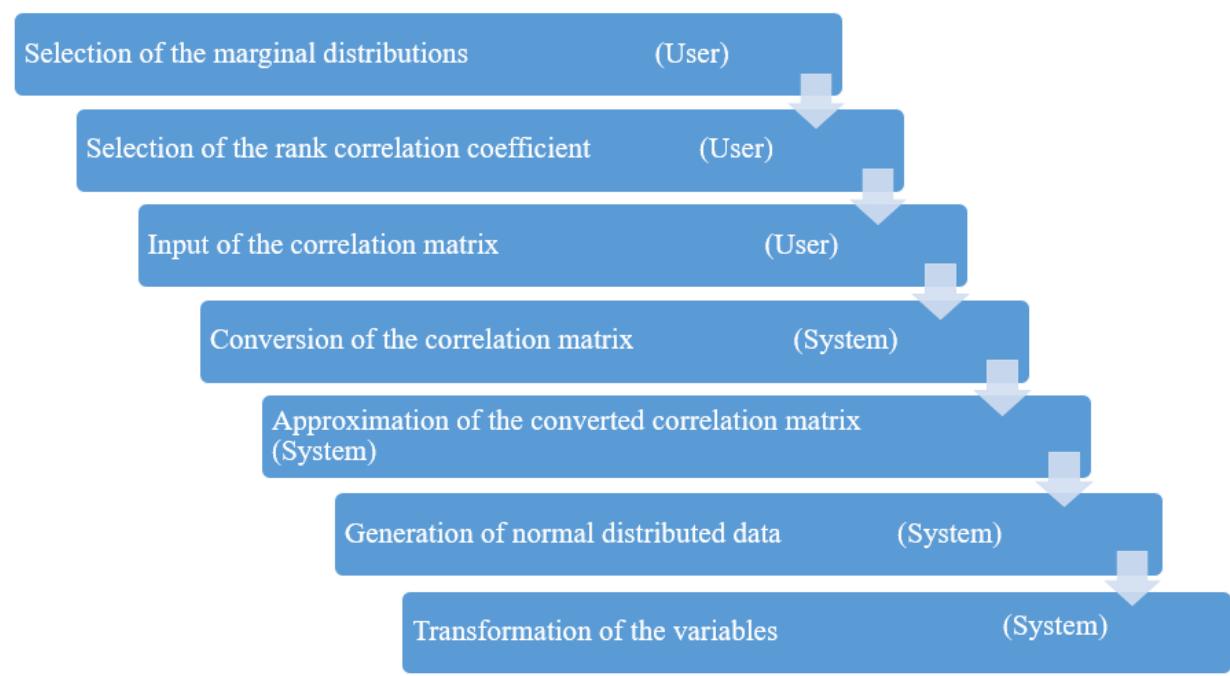

Figure 1. Process for generating the data set. 


\subsubsection{Selection of marginal distributions.}

First the marginal distributions of the individual variables are selected by the user. For each variable, theoretical considerations are made as to which distribution should be used. For example, the data can be normally distributed or log-normally distributed. Mixed distributions can also be considered.

\subsubsection{Selection of the rank correlation coefficient}

To show dependencies between the individual variables, the user specifies a correlation matrix. The user can choose between Spearman or Kendall coefficients. The specified values of the matrix correspond to the respective rank correlation coefficients. Spearman correlation is based on equal distances between the individual ranks. Kendall only considers the relative arrangement of the individual ranks and is rather used for smaller samples.

\subsubsection{Input of the correlation matrix}

For the input of the correlation matrix the permissible value range between -1 and 1 is given for the user. It is not necessary to enter all values if no specific correlation between two parameters is required. In this respect, missing values are permissible. The size of the matrix is determined by the number of selected parameters. A symmetric matrix is required for further calculations. To prevent errors, the entered values are mirrored at the main diagonal and entered automatically. After the input is complete, the individual coefficients must be converted, since Pearson correlation is required in an intermediate step to generate multivariate normal distributions.

\subsubsection{Conversion of the correlation matrix}

According to [15], two known formulas are used for the conversion depending on the selected rank correlation coefficient. The starting point is always the generation of data from the standard normal distribution. For very large data sets $>100$ entries, the correlation can be converted from Spearman $r_{s}$ to Pearson $r_{p}$ according Eq. (1). Eq. (2) is used to convert the correlation coefficients according to Kendall $r_{k}$.

$$
\begin{aligned}
& r_{p}=2 * \sin \left(\frac{\pi * r_{S}}{6}\right) \\
& r_{p}=\sin \left(\frac{\pi * r_{k}}{2}\right)
\end{aligned}
$$

After the conversion, the correlation coefficients according to Pearson can now be used to generate the data. For multivariate standard normal distributed data, a symmetric correlation matrix must be present which is positive semidefinite. To ensure this, the corrected correlation matrix is approximated to the original matrix in several steps.

\subsubsection{Approximation of the converted correlation matrix}

The approximation of the correlation matrix is divided into two steps. In the first step, the matrix has to fulfill certain requirements so that an optimization problem can be formulated to approximate this matrix. A matrix is positive semidefinite if it can be represented exclusively as a Gram matrix in Eq. (3). 


$$
M=V^{T} * V
$$

Here $V$ denotes any matrix and $V^{T}$ the transposed matrix of $V$. In the matrix $M$, correlations are given instead of covariances. Accordingly, the values must lie between -1 and 1 and must have the value 1 on the main diagonal. To ensure this condition, the columns $v_{1}, \ldots, v_{k}$ of matrix $V$ are normalized, so that $\left\|v_{i}\right\|=1$ applies. For the optimization problem, a corresponding positive semidefinite matrix is required, which is derived as initialization matrix from the matrix entered by the user.

In the second step an error function is defined which calculates the sum of the quadratic or absolute errors between the positive semidefinite matrix $\mathrm{M}$ and the entered matrix. As mentioned in chapter 3.1.3, the entered matrix may also contain erroneous values. Starting point is the initialization matrix and the original defined matrix. The error between the two matrices must be minimized. The required initialization matrix is created using a complex procedure. First the maximum absolute values outside the main diagonal are determined and the initialization matrix is adopted. Then the corresponding rows and columns are deleted and the same procedure is followed with the remaining matrix. All remaining matrix elements are initialized with 0 . All elements are mirrored after the main diagonal. An initialization matrix is available as a partially optimized matrix, which contains the largest correlations in terms of amount. In the last step the optimization method is selected. For a better overview, Figure 2 schematically illustrates the procedure for approximating the converted correlation matrix.

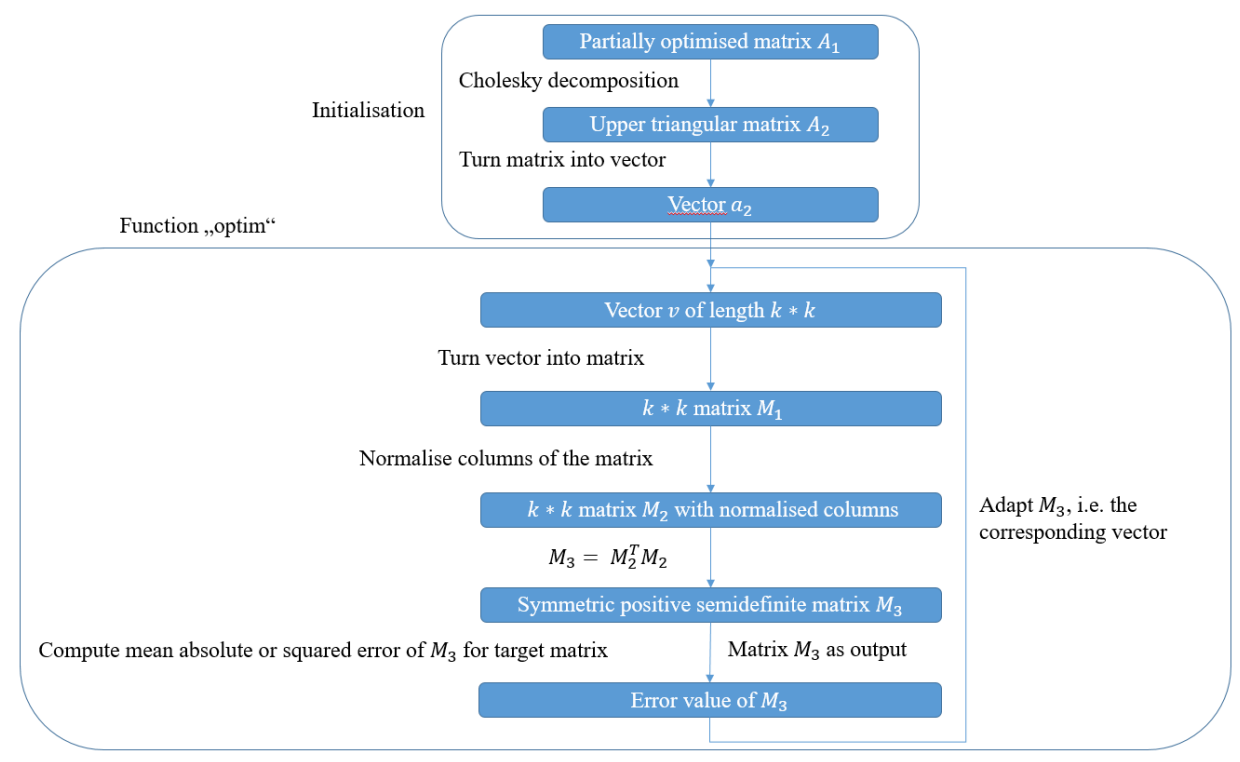

Figure 2. Procedure for approximating the converted correlation matrix.

In this paper the function "optim" in R was used. This function uses the NelderMead method for optimisation, a downhill simplex method. The method is used for the optimization of nonlinear problems, with several parameters. In addition to the defined objective function, the parameters for optimization are needed. These can be found in the already mentioned initialization matrix. The original matrix is then approximated with the help of the initialization matrix and the objective function [16]. This approximated matrix represents the result of the process. 


\subsubsection{Transformation of the data}

In the last step, the normally distributed data of the individual variables are transformed to the corresponding desired distributions. This is done by the inverse transformation method, a well-known method for generating pseudo-random numbers [17]. Given the values $x$ of a variable following a standard normal distribution, $\Phi(x)$ follows a uniform distribution on the unit interval where $\Phi$ denotes the cumulative distribution function of the standard normal distribution. If $F$ denotes the cumulative distribution function of the desired distribution then $F^{-1}(\Phi(x))$ follows - according to the inverse transformation method - the desired distribution. The inverse $F^{-1}$ of the cumulative distribution function or quantile function is computed numerically via a simple bisection method.

In principle, the data generator with direct use of the $\mathrm{R}$ functions can handle any continuous probability distribution offered by $\mathrm{R}$ and also any finite mixture of these distribution functions. The GUI-based version offers seven continuous distribution functions to choose from. In addition to the normal distribution, there is also the lognormal, the exponential, the $t-$, the $\chi^{2}$-, the beta and the gamma distribution. Different parameters must be specified for each distribution. Table 1 provides an overview of the parameters to be set for each distribution function.

Table 1. Overview of the parameters to be set for each distribution

\begin{tabular}{l|l}
\hline Distribution & Parameter to be set \\
\hline Normal distribution & Mean, sigma \\
\hline Log-normal distribution & Mean, sigma, shift \\
\hline Exponential distribution & Rate \\
\hline T distribution & Degrees of freedom of t \\
\hline Chi-square distribution & Degrees of freedom of chi-square \\
\hline Beta distribution & Shpae1, shape2 \\
\hline Gamma distribution & Shape gamma, shape rate \\
\hline
\end{tabular}

In addition to the individual distributions for transforming the data, a mixed distribution can also be selected. From the distribution functions shown in Table 1, any finite mixture can be generated directly in $\mathrm{R}$. The advantageous direct use is the increased flexibility for combining the individual distributions. The use of this possibility with the graphical user interface described in Section 5.3 is, however, clearly limited. Therefore, up to five different normal distributions with different values for the parameters mean and standard deviation can be combined for a mixture. The proportion of each individual distribution in the mixed distribution can be varied.

\subsection{Insertion of outliers}

After a possible transformation of the variables, outliers can also be included. Depending on the number of selected variables, exactly one outlier can be inserted into each record. To do this, the current maximum value of the corresponding variables is determined and increased by $25 \%$. The rank correlation is only slightly changed by an outlier and thus remains almost unchanged. The data set is then available as a CSV file for the selected variables based on a correlation matrix, the use of a transformation and the insertion of outliers. 


\section{Studies on correlation coefficients and matrix approximation}

In this chapter, we examine which of the correlation coefficients according to Spearman or Kendall is better suited for the initial correlation matrix. Furthermore, different initialization and optimization strategies for the approximation of the given but possibly inconsistent correlation matrix are compared.

\subsection{Difference between Spearman and Kendall correlation to Pearson correlation}

As already mentioned, the desired correlations between variables are given in the form of a rank correlation coefficient, since these are more meaningful to interpret in the case of skewed distributions and existing outliers than the Pearson correlation. To generate multivariate data, a multivariate normal distribution is first used. Those marginal distributions are then transformed into the correspondingly specified marginal distributions. Therefore, Pearson correlations are required for the covariance matrix of the multivariate normal distribution. As explained in Section 3.1.4, the rank correlation coefficients for normal distributions can be converted to Pearson correlations.

Figure 3 shows on the $\mathrm{x}$-axis the value ranges for the rank correlation coefficients according to Spearman and Kendall. The y-axis indicates the absolute difference to the corrected value of the correlation coefficient according to Person. The Kendall correlation - represented by the blue line - shows significantly larger deviations from the Pearson correlation than the Spearman correlation (black line).

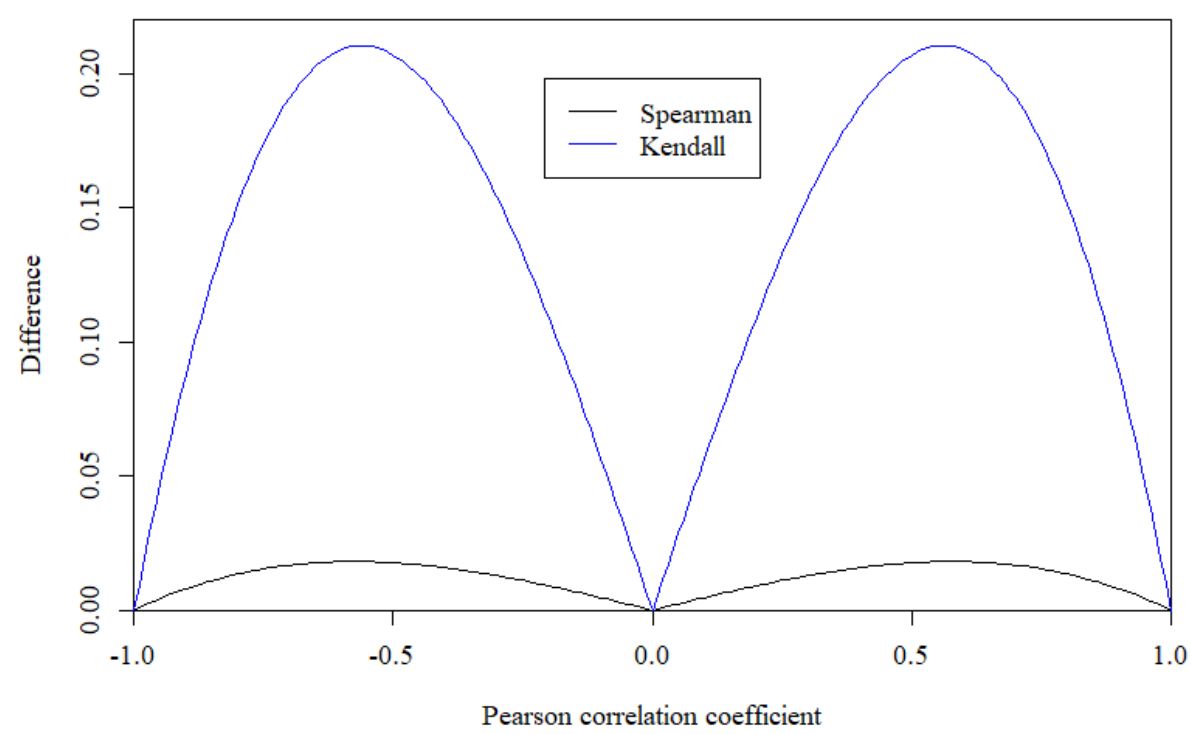

Figure 3. Difference between the two rank coefficients and the Pearson correlation coefficient.

The average deviation in the conversion is 0.012 for Spearman and 0.13 for Kendall. At the points -0.58 and 0.58 the maximum difference is 0.018 for Spearman and 0.21 for Kendall at the points -0.56 and 0.56 . The generated data will always show random deviations from the given correlation coefficients. This deviation is scaled accordingly 
by the required conversion, so that when using the Kendall correlation, the deviations are significantly larger than with the Spearman correlation. Spearman correlation should therefore generally be used.

\subsection{Selection of the initialization matrix}

For the matrix approximation, a target or error function is defined that is to be minimized. Here we use either the mean squared error or the mean absolute error. The optimization procedure requires a starting value, i.e. an initial matrix must be specified. In order to achieve the best possible approximation, three possibilities for creating the initialization matrix are examined: the matrix with all entries equal to 1 , the identity matrix and the matrix generated by the strategy mentioned in Section 3.1.5 in which the absolute maximum values of each row or column of the entered matrix are selected and all other matrix elements are initialized with 0 .

In order to judge the influence of the selected initialization matrix, the mean squared error $e$ between the original matrix $A=\left(a_{i j}\right)$ and the approximated matrix $B=\left(b_{i j}\right)$ is calculated as an error measure. However, other error measures are also possible. The smaller this error is, the more suitable the selected initialization matrix is for approximation by means of the optimization procedure. To evaluate the approximation errors, we generated random matrices with two different methods. Firstly, random symmetric matrices with values in the interval $[-1,1]$ from a uniform distribution are generated. The elements on the main diagonal are set to 1 and the symmetry is enforced. Such a matrix will in general not be positive semidefinite. As a second test, random positive semidefinite matrices are generated. The generation of such a matrix was already explained in Section 3.1.5. The matrix elements for the matrix $V$ in Eq. (3) are generated from a normal distribution. In both cases, missing values $n a$ are randomly inserted. The probability $p$ for missing values depends on the size $n$ of the matrix. In the various experiments, the rank correlation matrix according to Spearman is always used for the generated correlation matrix. Kendall correlation is not considered due to the reasons explained in Section 4.1. The matrix random matrix is converted in terms of Spearman correlations and is then approximated. For the present investigations, different sizes of the symmetric matrix are tested with a variable probability for missing values. For a matrix size of 4 the probability is $0 \%$, for a size of $850 \%$ and for a size of $1670 \%$. The idea behind the increase of the probability for missing values with the size of the matrix is that for large number of variables one would not expect a user to specify target correlations for all pairs of the variables. A total of 100 simulations are performed for each generated matrix and the mean squared error is calculated over all non-missing values outside the diagonal. In the following section, the three possibilities for matrix initialization are presented.

\subsubsection{Matrix of ones}

The first variant is to use the matrix of ones as initialization matrix for the optimization procedure. For 100 simulations, depending on the two methods and the size of the matrix, the following Figures 4 and 5 show the distribution of the errors. 


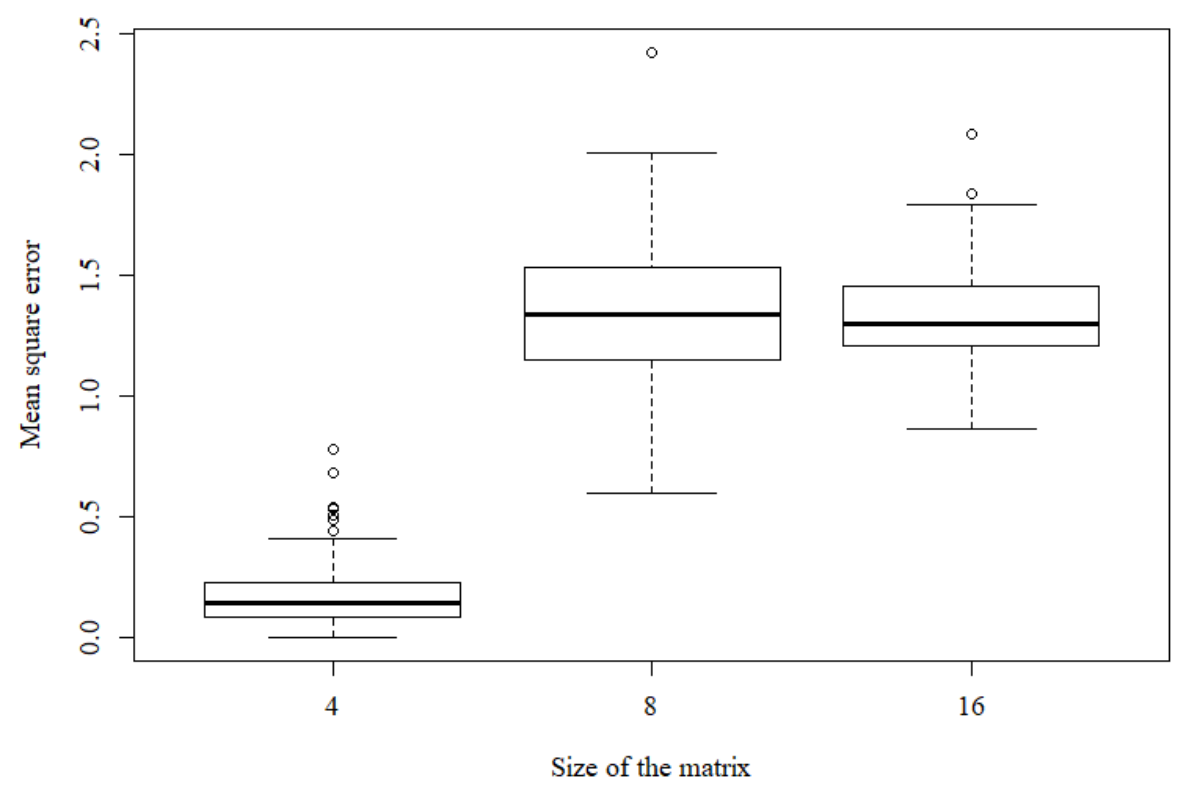

Figure 4. Boxplots for displaying the error as a function of the matrix size when using the matrix of ones for initialization to approximate a random symmetric matrix.

Figure 4 shows that for randomly generated symmetric matrices, the error also increases with increasing matrix size. The more missing values are present, the lower the probability that the (partial) matrix is inconsistent, so that a better approximation is possible. The mean squared error is too large to be acceptable. 


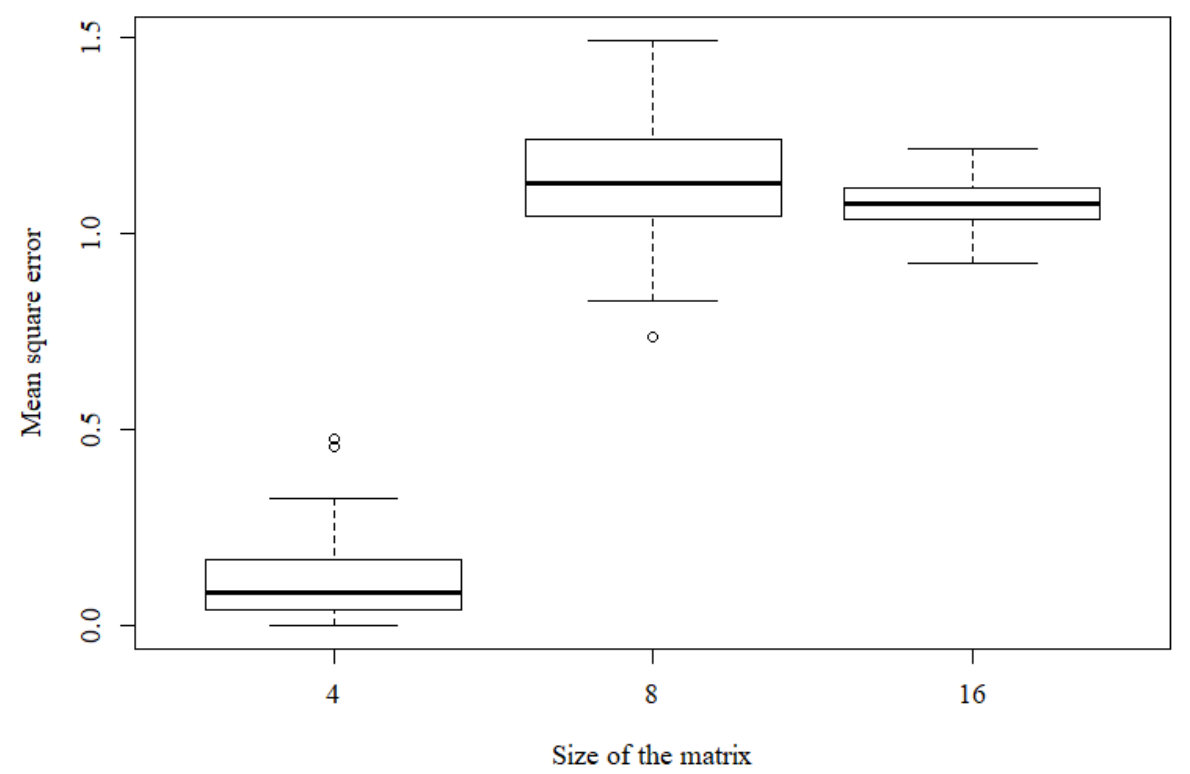

Figure 5. Boxplots for displaying the error as a function of the matrix size when using the matrix of ones for initialization to approximate a random positive semidefinite matrix.

Figure 5 shows the corresponding results for approximating randomly generated positive semidefinite matrices. The results are slightly better compared to Figure 4 because the matrices to be approximated are already positive semidefinite. But the error is still not acceptable.

\subsubsection{Identity matrix}

As a second variant for the initialization matrix of the optimization procedure, all matrix elements on the main diagonal of the identity matrix are assigned the value 1 and all other elements 0 . 


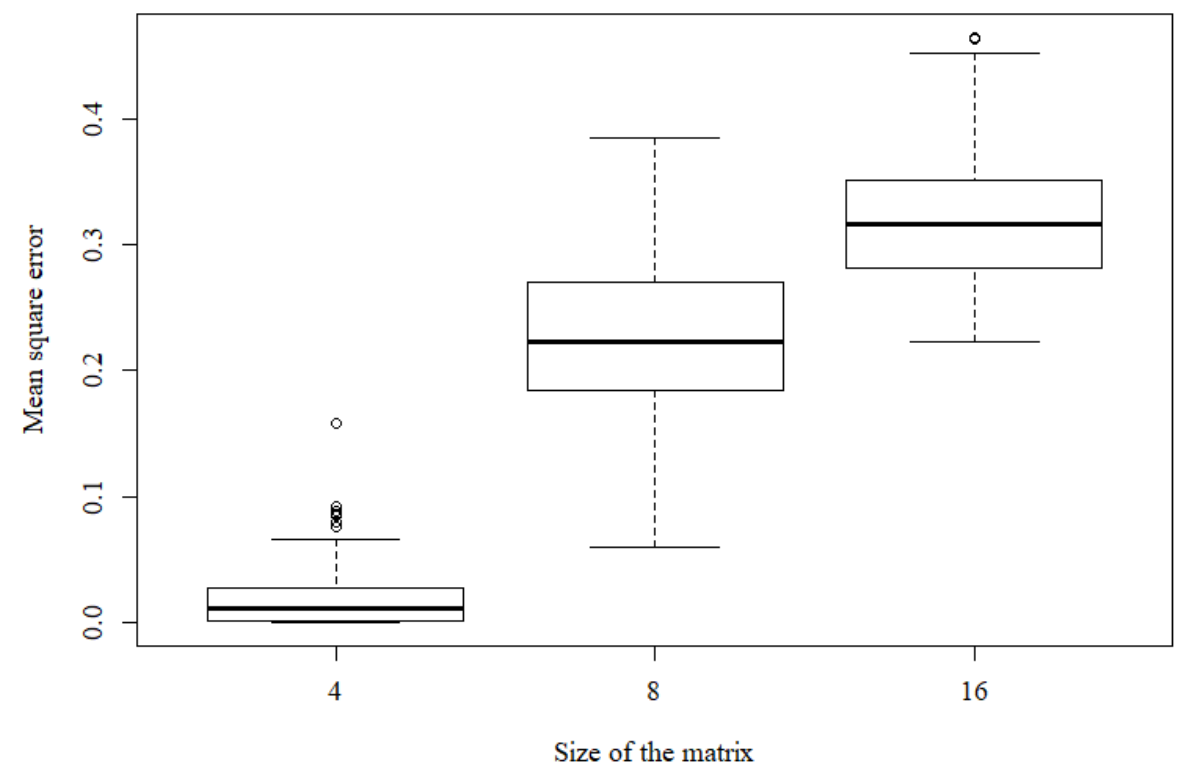

Figure 6. Boxplots for displaying the error as a function of the matrix size when using the identity matrix for initialization to approximate a random symmetric matrix.

Figure 6 shows that for randomly generated symmetric matrices, the error and thus the median also increases with increasing matrix size. Outliers can be seen with matrix sizes 4 and 16 . The scattering width is greatest with a matrix size of 8 . 


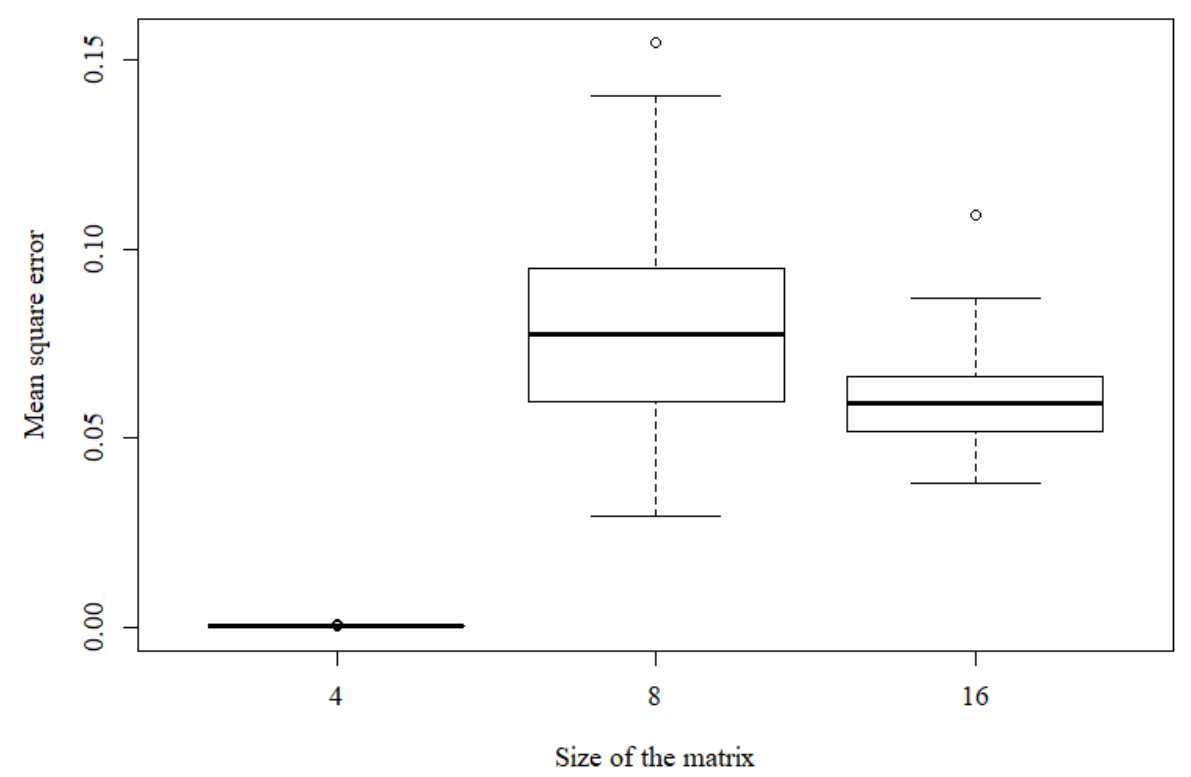

Figure 7. Boxplots for displaying the error as a function of the matrix size when using the identity matrix for initialization to approximate a random positive semidefinite matrix.

Figure 7 shows that the error and thus the median for randomly generated positive semidefinite matrices is significantly smaller than for randomly generated symmetric matrices. The scattering width is also smaller for all three matrix sizes. However, the few identified outliers at a size of 8 are still smaller than, for example, the median at identical size for a randomly symmetric generated matrix.

Compared to the matrix of ones, the error in choosing the identity matrix as initialization matrix is significantly smaller for both methods. Therefore, the identity matrix as initialization matrix is more suitable for approximation than the matrix of ones.

\subsubsection{Initialization using a partially optimized matrix}

The third variant does not use a predefined matrix, but the initialization matrix as a partially optimized matrix. The complex procedure has already been explained in chapter 3.1.5. This matrix contains the maximum absolute values outside the main diagonal of the original matrix. All other matrix elements are initialized with 0 . 


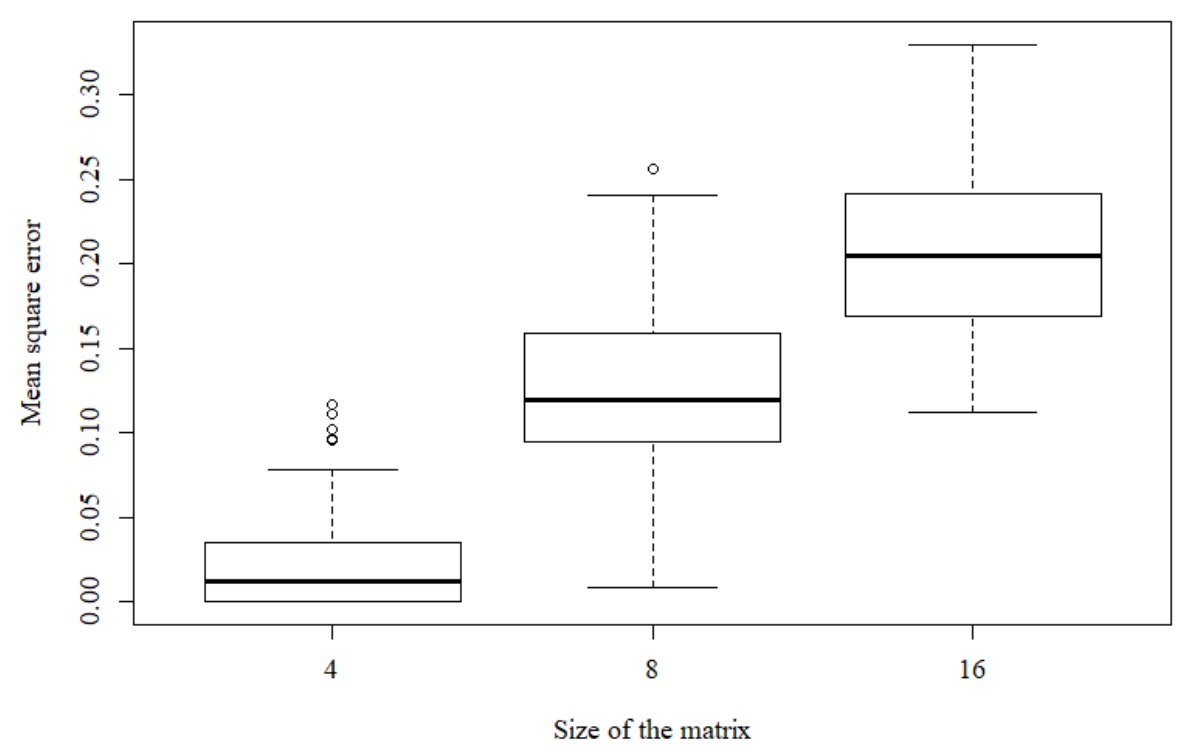

Figure 8. Boxplots for displaying the error as a function of the matrix size when using the partially optimized matrix for initialization to approximate a random symmetric matrix.

Figure 8 shows that even when using the partially optimized matrix as initialization matrix, the error increases with increasing matrix size for randomly symmetrical matrices. The median therefore also increases. The initialization with the partially optimized matrix is obviously superior to the two other initialization methods. 


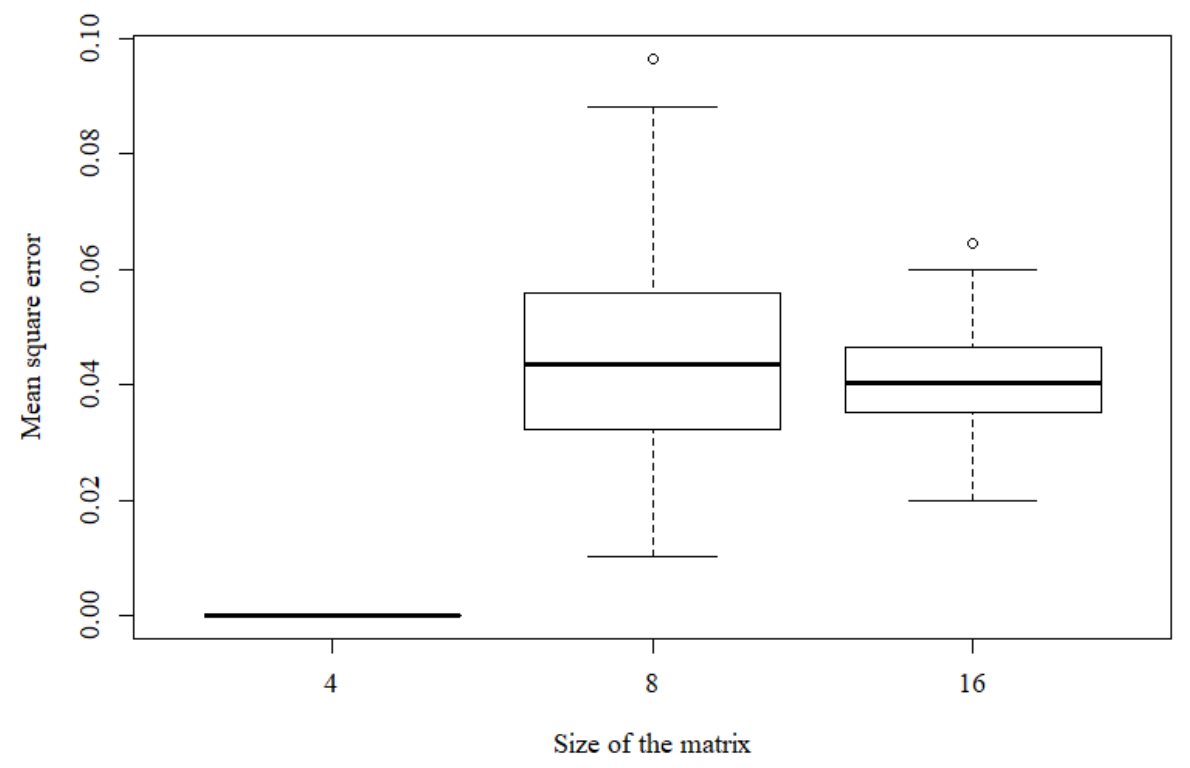

Figure 9. Boxplots for displaying the error as a function of the matrix size when using the partially optimized matrix for initialization to approximate a random positive semidefinite matrix.

Figure 9 shows a similar result when the partially optimized matrix is used for initialization to approximate a random positive semidefinite matrix. The error is smaller as even smaller compared to the approximation of a random matrix due to the fact the matrix to be approximated is already consistent in terms of being positive semidefinite.

Overall, the use of the partially optimized matrix leads to the best result for the initialization matrix, regardless of the method for generating random matrices. Even with the random symmetric matrices, this method gives the best result. For random positive semidefinite matrices, the average error of 0.05 is almost negligible. This means that the random matrix can be approximated very well with this method, or the matrix can be reproduced almost directly by the approximation due to the positive semi definiteness. Accordingly, the defined correlation properties are not lost in the conversion and subsequent approximation. The large number of missing values at a matrix size of 16 leads to an improved approximation due to a lower inconsistency of the (partial) matrix. The other two possibilities for matrix initialization are not relevant for the rest of the process. For the data generator, the partially optimized matrix should therefore be used to generate the initialization matrix, as already explained in chapter 3.1.5.

\subsection{Comparison to a method by NJ Higham for approximating a positive semidefinite matrix}

NJ Higham [18] developed an algorithm to calculate the closest symmetric positive semidefinite matrix in the Frobenius norm to any given matrix $A$. Higham's method was not designed for coping with missing values. Therefore, we set all missing values in the 
matrix to be approximated to 0 . For the mean squared error for comparison to our method, these positions are not counted.

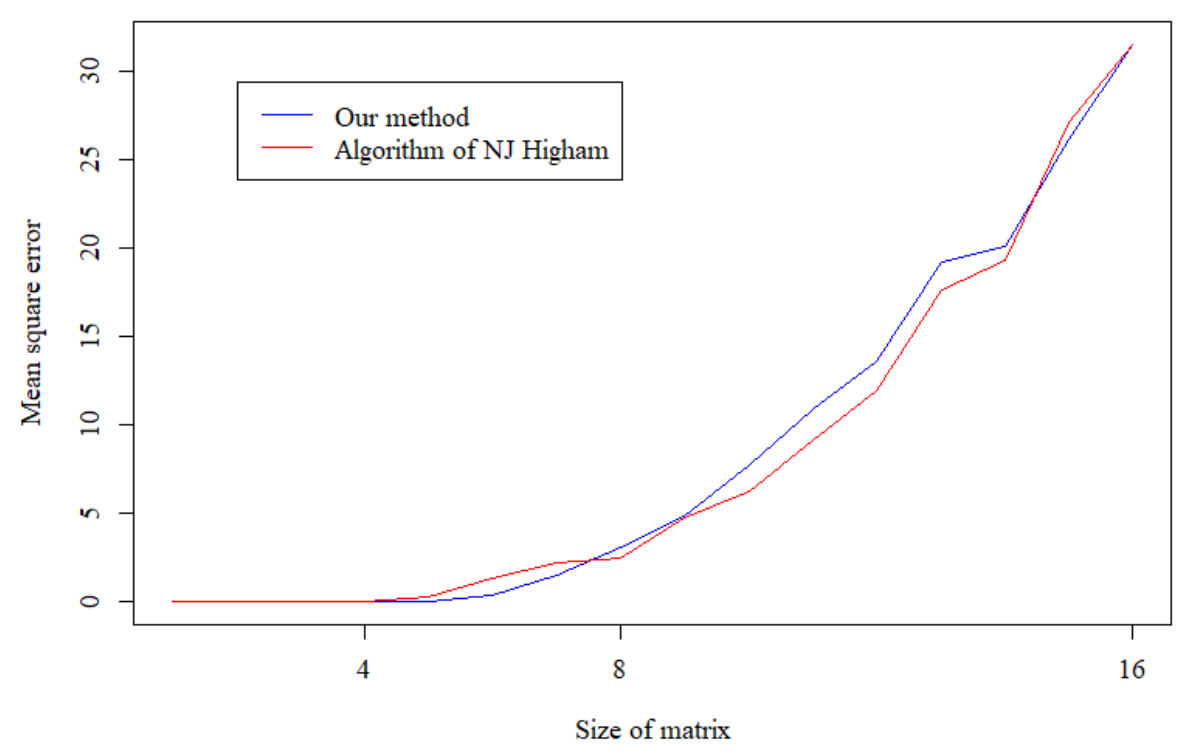

Figure 10. Comparison of the error between our and NJ Higham's method to approximate a random symmetric matrix by a positive semidefinite matrix depending on the matrix size.

Figure 10 shows the error as a function of the matrix size for our and NJ Higham's method. Overall, the error of our method is always smaller for small matrices up to a size of 7. For large matrices, the error of the algorithm is somewhat smaller, but for a matrix size of 16, both errors are approximately identical.

\section{Example for using the data generator}

Data sets from application and research projects may often not be used in teaching for reasons of confidentiality. However, real data sets sometimes contain interesting patterns and properties. Instead of the real data set, an artificial data set can be created with the presented method. It has statistically similar properties to the real data set and can be used in teaching without any problems. In Section 3 the steps to generate artificial data by a correlation matrix and different distribution functions were presented. The exact procedure is demonstrated in this section using the iris data set [19]. This data set was created in 1935 as part of the investigation of irises. In total, the data set contains measured data of 150 iris plants. These are divided into 50 Virginia irises (Iris Virginica), bristly irises (Iris setosa) and irises of different colours (iris versicolor). The length and width of the sepals and petals of each individual plant are included. If this data set would not contain information about flowers but about patients or internal data of a company, it should not be used in teaching. The iris data set will be considered here without the categorical attribute species. In order to generate a random data set with similar 
properties to the iris data set, the marginal distributions of the individual attributes and the Spearman correlation matrix are required. From the Spearman correlation the corresponding Pearson correlations are computed according to Eq. (1). The Pearson correlation matrix might not be positive semidefinite because it was not directly calculated from the data but indirectly via the Spearman correlations. Then data from a corresponding multivariate normal distribution with the approximated Pearson correlation matrix are generated. Then the normally distributed data in the individual dimensions are transformed so that distributions similar to those in the original data set are obtained. How the transformations are determined is described in Section 5.2. Afterwards, the data are available in an anonymized form with nearly the same original properties of the original data set. For the general data generation, the data generator with a graphical user interface can be used.

\subsection{Determination of the correlation matrix}

The data generator uses as input the correlation matrix of the iris data set according to Spearman. The entire procedure for converting the correlation matrix and subsequent approximation was explained in Section 3.1. For illustration purposes, five tables are presented. For the calculation the class attribute (plant species) was removed from the data set. Table 2 shows the Spearman correlations of the original iris data set.

Table 2. Spearman correlations of the iris data set.

\begin{tabular}{l|l|l|l|l}
\hline & Sepal.Length & Sepal.Width & Petal.Length & Petal.Width \\
\hline Sepal.Length & 1.000 & -0.167 & 0.882 & 0.834 \\
\hline Sepal.Width & -0.167 & 1.000 & -0.310 & -0.289 \\
\hline Petal.Length & 0.882 & -0.310 & 1.000 & 0.938 \\
\hline Petal.Width & 0.834 & -0.289 & 0.938 & 1.000 \\
\hline
\end{tabular}

Then the Spearman correlation is converted into Pearson correlations according to chapter 3.1.4 and the matrix with the Pearson correlations is approximated by a positive semidefinite matrix. Note that the matrix with the Pearson correlations obtained by converting the Spearman correlations does not necessarily have to be positive semidefinite. Table 3 shows the approximated Pearson correlations for the iris data set.

Table 3. Approximated Pearson correlations of the iris data set.

\begin{tabular}{l|l|l|l|l}
\hline & Sepal.Length & Sepal.Width & Petal.Length & Petal.Width \\
\hline Sepal.Length & 1.000 & -0.176 & 0.890 & 0.848 \\
\hline Sepal.Width & -0.176 & 1.000 & -0.321 & -0.301 \\
\hline Petal.Length & 0.890 & -0.321 & 1.000 & 0.943 \\
\hline Petal.Width & 0.848 & -0.301 & 0.943 & 1.000 \\
\hline
\end{tabular}

For comparison, Table 4 shows the original Pearson correlations of the iris data set.

Table 4. Original Pearson correlations of the iris data set calculated from the data.

\begin{tabular}{l|l|l|l|l}
\hline & Sepal.Length & Sepal.Width & Petal.Length & Petal.Width \\
\hline Sepal.Length & 1.000 & -0.118 & 0.872 & 0.818 \\
\hline Sepal.Width & -0.118 & 1.000 & -0.428 & -0.366 \\
\hline Petal.Length & 0.872 & -0.428 & 1.000 & 0.963 \\
\hline Petal.Width & 0.818 & -0.366 & 0.963 & 1.000 \\
\hline
\end{tabular}

The result of the correlation matrix approximation is almost identical to the original matrix. This ensures that the correlation properties are preserved for the transformation of the data. With this correlation matrix 150 records are generated from a 4-dimensional 
centred normal distribution. Table 5 shows the Spearman correlations of the generated data set.

Table 5. Spearman correlations of the generated data set.

\begin{tabular}{l|l|l|l|l}
\hline & Sepal.Length & Sepal.Width & Petal.Length & Petal.Width \\
\hline Sepal.Length & 1.000 & -0.144 & 0.907 & 0.847 \\
\hline Sepal.Width & -0.144 & 1.000 & -0.208 & -0.183 \\
\hline Petal.Length & 0.907 & -0.208 & 1.000 & 0.943 \\
\hline Petal.Width & 0.847 & -0.183 & 0.943 & 1.000 \\
\hline
\end{tabular}

These correlation values are then converted again. Table 6 shows the Pearson correlations of the generated data set.

Table 6. Pearson correlations of the generated data set.

\begin{tabular}{l|l|l|l|l}
\hline & Sepal.Length & Sepal.Width & Petal.Length & Petal.Width \\
\hline Sepal.Length & 1.000 & -0.151 & 0.912 & 0.864 \\
\hline Sepal.Width & -0.151 & 1.000 & -0.240 & -0.224 \\
\hline Petal.Length & 0.912 & -0.240 & 1.000 & 0.949 \\
\hline Petal.Width & 0.864 & -0.224 & 0.949 & 1.000 \\
\hline
\end{tabular}

The correlation matrices of the generated data set show that the original correlations are essentially preserved. In the next step, the marginal data are then transformed to obtain the distributions similar to the original ones.

\subsection{Transformation of the data}

With the transformation of the data, the marginal distributions of the individual variables are approximated. The data generator needs a specification of the desired marginal distributions. Because we have real data here, we derive the marginal distributions from the data by approximating them with finite Gaussian mixture models. These models have been adapted using an EM algorithm for model-based clustering, classification and density estimation [20]. Here we use the package mclust provided in R to determine the corresponding mixture models. For the variables Sepal.Length and Sepal.Width, mclust approximates the data with just one normal distribution each. The mixture models for the other two variables, Petal.Length and Petal.Width, consist each of a mixed distribution of two normal distributions. The corresponding distributions can be defined in the graphical user interface or directly in R. Then the inverse transformation method is applied to generate the desired marginal distributions from the normal distributions.

The result is a data set that is very similar to the original iris data set and, in addition to the properties of the correlation matrix, also has the properties of the respective marginal distribution. In order to be able to compare both data sets with each other, the original and the artificially generated data set is represented by a respective scatter plot over the four variables in Figures 12 and 13. 


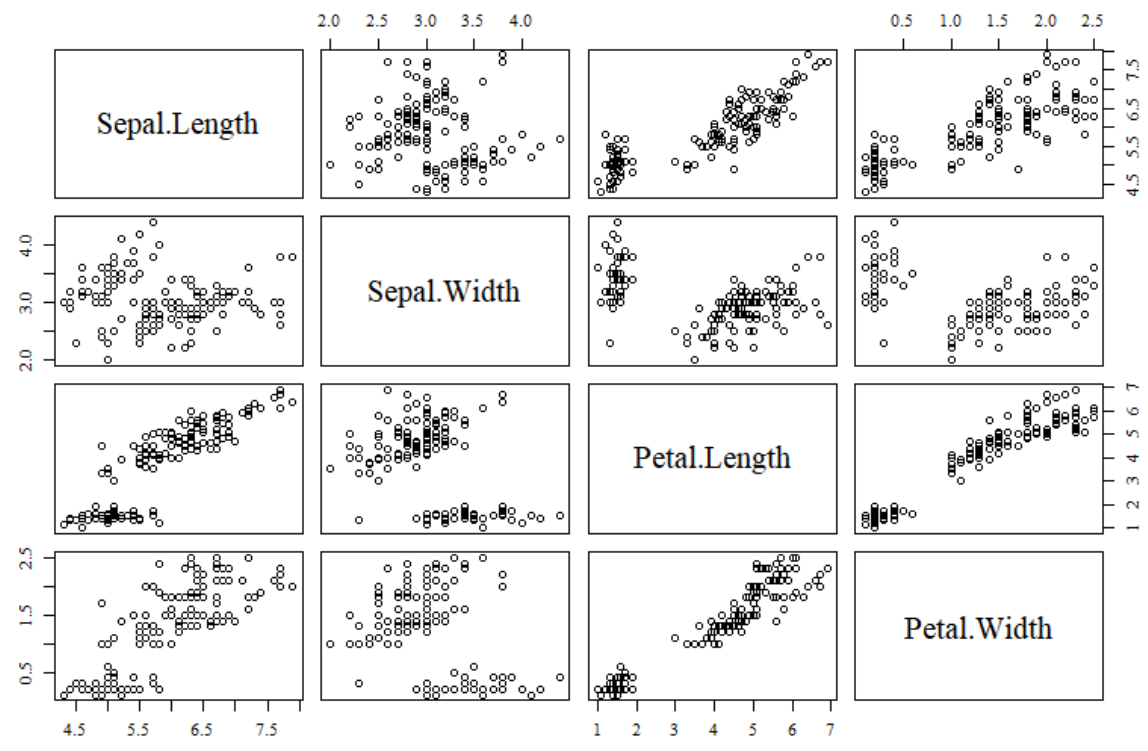

Figure 11. Scatter plot of the original iris data set.

Figure 11 shows the scatter matrix of the original iris data set.

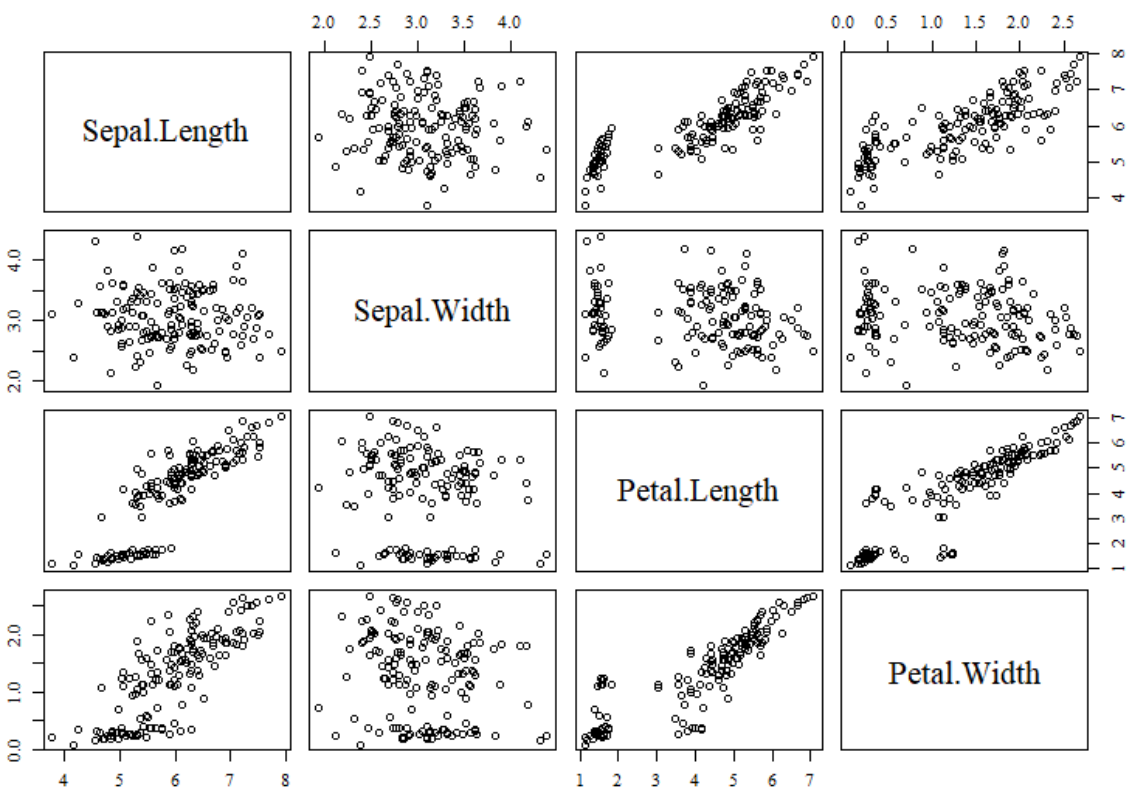

Figure 12. Scatter plot of the generated iris data set.

Figure 12 shows the generated "fake" iris data set. This data set is very similar to the original data set. However, the data are not exactly the real ones, only their properties 
were taken into account. Since random values were generated from a normal distribution, there are certain deviations on some sub-images of the scatter plot. Nevertheless, this artificial iris data set can be used for further investigations if the real data must not be used. Accordingly, the developed data generator is suitable for the generation of artificial data taking into account the real properties and relationships between the individual variables.

\subsection{Design of the graphical user interface}

The data generator offers the possibility to easily generate the desired data with the corresponding properties. The basis for the graphical user interface is the package "Shiny" in R. It allows the creation of interactive web applications. Either on the local computer or integrated into a dashboard. The package combines the modern interactivity of the web with the computing power of R. Large amounts of data can be evaluated in different ways and the results can be displayed clearly. This facilitates the interpretation for the corresponding tasks [21]. Figure 13 shows the three areas of the graphical user interface of the data generator.

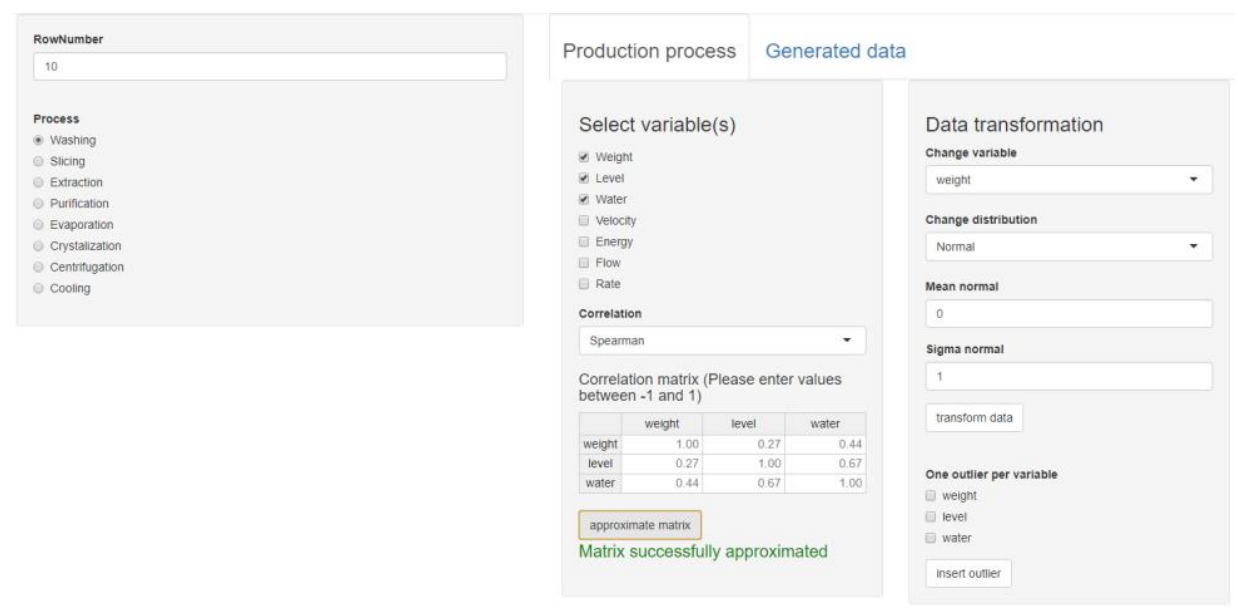

Figure 13. Graphical user interface of the data generator

The generation of the data is based on an exemplary process from the industry. This process is divided into several sub-steps, which are not examined in detail. First, the size of the data set to be generated is determined based on the number of rows. Then the desired process step is selected. Each of the sub-steps has different parameters. Depending on the settings of the data generator, one or more parameters for generating the data set can be selected under "Production Process". In addition, the relationships between the parameters can be defined using a correlation matrix. After the matrix has been approximated, a first data set according to a multivariate normal distribution is available under "Generated Data". In the next step, the individual variables can still be transformed according to certain distribution functions and outliers can be inserted. The finished data set is available at the end as a CSV file and can be used in many programs for evaluation and analysis.

In the GUI, the selection of distributions is limited. In R, any number of mixtures of continuous distributions can be generated directly. The following $\mathrm{R}$ Code shows an example. 


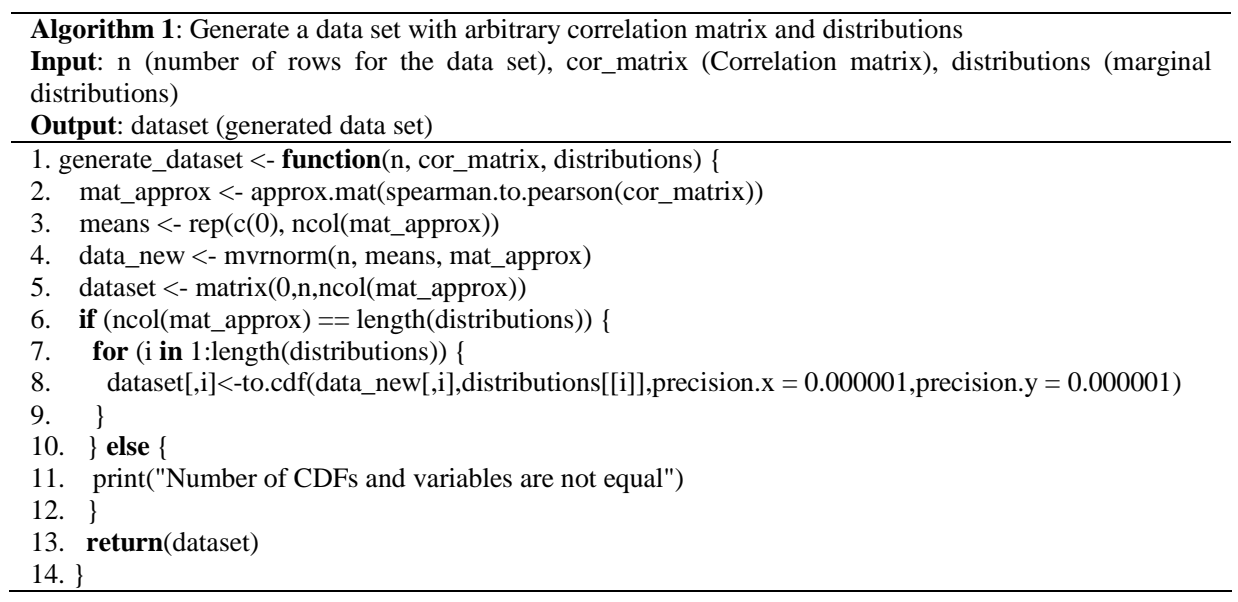

The function needs a number of rows of the data set, the correlation matrix and a vector of distributions. This can be mixtures of continuous distributions or individual distributions. All available distributions are shown in Table 1. The used functions are developed for the data generator.

\section{Conclusion}

Data generators for generating artificial data offer many advantages. Since real data are either not accessible for certain questions or - for example for benchmark or test purposes - certain properties should be present in a data set, artificial data must be used. An advantage for real data is that no one can argue against it, because the characteristics of the data depend on a real process. Due to data protection reasons, it may not always be possible to use real data so easily. Artificial data, on the other hand, have a known content that fulfils certain conditions but is not real. Furthermore, the data should be derived from a real problem in order to establish a connection to reality.

This paper has shown how artificial data can be generated using a data generator developed for this purpose. Within this data, certain effects can be included. The data generator is very flexible and can be used in many application areas. The graphical user interface is easy and intuitive to use. Especially the many possibilities to use different correlations and distribution functions for the data represents the great individual use of the data generator. A practical example was also used to show that properties of real data can also be adopted and incorporated into the artificial data. In this respect, real data can be made anonymous and then used without any problems. Furthermore, sometimes existing data have to be supplemented or replaced with artificial data. In this case, values may be below the level of detection (LOD). Procedures are described in [22] and [23] that can replace these missing values. The missing or to be replaced data are estimated and replaced according to a certain distribution.

In the future, the data generator can be extended by further features. For example, the possibility of generating data that is also time-dependent. In addition, the option of generating categorical data can be further extended. In its current form, the data generator can already be used for individual categorical data. For example, when generating an artificial iris data set as described in chapter 5, a separate data set could have been 
generated for each value in the domain of the categorical attribute and these individual data sets including the categorical attribute could have been merged into one complete data set.

The integration of outliers can also be extended, e.g. to increase the number of outliers per variable. The same applies to the distribution functions for transforming the variables. Especially the mixed distributions consisting of different distributions can be interesting. If the graphical user interface is not used, this is already possible with the Rfunctions provided. In the graphical interface, only several normal distributions can currently be combined with each other. A further development of the data generator can lead to an even higher flexibility and individualization of the data. At the same time, other effects can be incorporated. In this respect, the complexity of the data set can be further extended. By improving the data generator, a holistic approach can be pursued in the future. It may then no longer be necessary to use a separate data generator for each specific question. The entire data generator including the graphical user interface can be downloaded from GitHub [24].

\section{Acknowledgments}

This research has been funded by the Lower Saxony Ministry of Science (Funding reference number: Innovation plus (2019/2020), No. 43) and the Federal Ministry of Economic Affairs and Energy (Funding reference number: 03ET1624C).

Supported by:

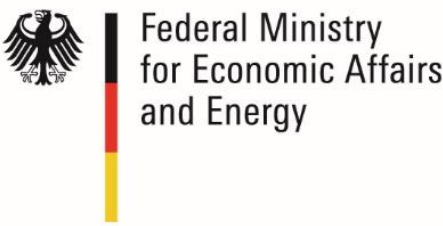

on the basis of a decision

by the German Bundestag

\section{References}

[1] K.-M. Osei-Bryson and C. Barclay, Knowledge Discovery Process and Methods to Enhance Organizational Performance, CRC Press, Boca Raton, 2015.

[2] Y. Pei and O. R. Zaïane, Synthetic Data Generator for Clustering and Outlier Analysis Technical report, 2006.

[3] D. A. Rachkovskij and E. M. Kussul, DataGen: a generator of datasets for evaluation of classification algorithms, In: Pattern Recognition Letters 19(7) (1998), 537-544.

[4] B. Bischl, J. Schiffner and C. Weihs, Benchmarking Classification Algorithms on High-Performance Computing Clusters, In: Studies in Classification, Data Analysis, and Knowledge Organization, Springer, Heidelberg, 2014.

[5] O. V. Demler, M. J. Pencina and R. B. D'Agostino Sr., Impact of correlation on predictive ability of biomarkers, In: Statistics in Medicine 32(24) (2013), 4196-4210. 
[6] Z. Wang, P. Myles and A. Tucker, Generating and Evaluating Synthetic UK Primary Care Data: Preserving Data Utility \& Patient Privacy, In: 2019 IEEE 32nd International Symposium on Computer-Based Medical Systems (CBMS) (2019), 126-131.

[7] Y. Park and J. Ghosh, PeGS: Perturbed Gibbs Samplers that Generate Privacy-Compliant Synthetic Data, In: Transactions on Data Privacy 7(3) (2014), 253-282.

[8] J. Drechsler, Using support vector machines for generating synthetic datasets, International Conference on Privacy in Statistical Databases, Springer, Berlin, Heidelberg, 2010.

[9] F. Iglesias, T. Zseby, D. Ferreira and A. Zimek, MDCGen: Multidimensional Dataset Generator for Clustering, In: Journal of Classification 36 (2019), 599-618.

[10] H. Demirtas, A method for multivariate ordinal data generation given marginal distributions and correlations, In: Journal of Statistical Computation and Simulation 76(11) (2006), 1017-1025.

[11] I. Adä and M. R. Berthold, The New Iris Data: Modular Data Generators, In: KDD'10: Proceedings of the 16th ACM SIGKDD international conference on Knowledge discovery and data mining (2010), 413422.

[12] K. Pearson, Notes on regression and inheritance in the case of two parents, In: Proceedings of the Royal Society of London 58 (1895), 240-242.

[13] C. Spearman, The proof and measurement of association between two things, In: The American Journal of Psychology 15(1) (1904), 441-471.

[14] M. G. Kendall, A new measure of rank correlation, In: Biometrika 30(1-2) (1938), 81-93.

[15] W. Xu, Y. Hou, Y. S. Hung and Y. Zou, Comparison of Spearman's rho and Kendall's tau in Normal and Contaminated Normal Models, In: Signal Processing 93(1) (2013), 261-276.

[16] J. A. Nelder and R. Mead, A Simplex Method for Function Minimization, In: The Computer Journal 7(4) (1965), 308-313.

[17] L. Devroye, Non-Uniform Random Variate Generation, Springer, New York, 1986.

[18] N. J. Higham, Computing a nearest symmetric positive semidefinite matrix, In: Linear Algebra and its Applications 103 (1988), 103-118.

[19] T. A. Runkler, Data Analytics: Models and Algorithms for Intelligent Data Analysis, Springer, Wiesbaden, 2012.

[20] L. Scrucca, M. Fop, T. B. Murphy and A. E. Raftery, mclust 5: clustering, classification and density estimation using Gaussian finite mixture models, In: The R Journal 8(1) (2016), 205-233.

[21] R Core Team, R: A language and environment for statistical computing. R Foundation for Statistical Computing, URL https://www.R-project.org/, 2019.

[22] H. Chen, S. A. Quandt, J. G. Grzywacz and T. A. Arcury, A Distribution-Based Multiple Imputation Method for Handling Bivariate Pesticide Data with Values below the Limit of Detection, In: Environmental Health Perspectives, 119(3) (2011), 351-356.

[23] O. Harel, N. Perkins, and E. F. Schisterman, The Use of Multiple Imputation for Data Subject to Limits of Detection, In: Sri Lankan Journal of Applied Statistics, 5(4) (2014), pp.227-246

[24] https://github.com/kvahldiek/data_generator 